\title{
A Time-Dependent Buoyant Puff Model for Explosive Sources
}

\author{
Edward J. Kansa
}

\section{October 1997}

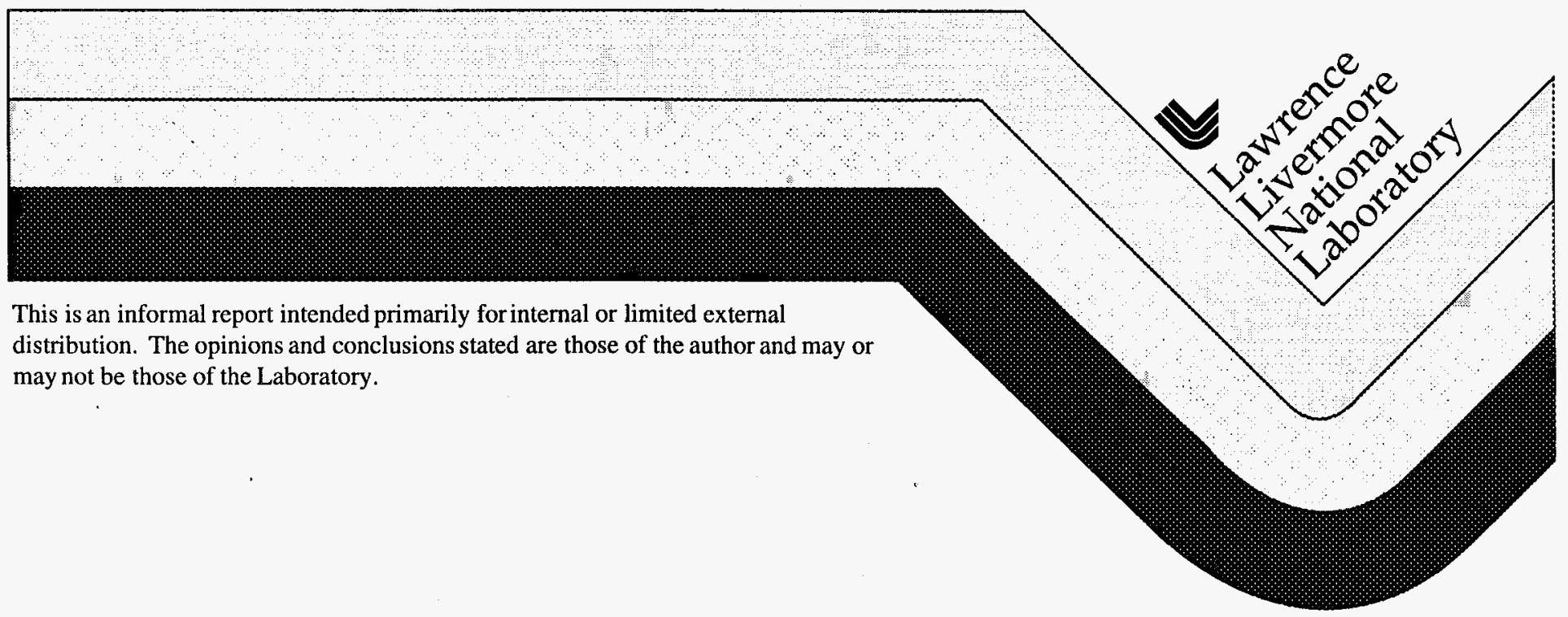




\section{DISCLAIMER}

This document was prepared as an account of work sponsored by an agency of the United States Government. Neither the United States Government nor the University of California nor any of their employees, makes any warranty, express or implied, or assumes any legal liability or responsibility for the accuracy, completeness, or usefulness of any information, apparatus, product, or process disclosed, or represents that its use would not infringe privately owned rights. Reference herein to any specific commercial product, process, or service by trade name, trademark, manufacturer, or otherwise, does not necessarily constitute or imply its endorsement, recommendation, or favoring by the United States Government or the University of California. The views and opinions of authors expressed herein do not necessarily state or reflect those of the United States Government or the University of California, and shall not be used for advertising or product endorsement purposes.

This report has been reproduced directly from the best available copy.

Available to DOE and DOE contractors from the Office of Scientific and Technical Information P.O. Box 62, Oak Ridge, TN 37831

Prices available from (615) 576-8401, FTS 626-8401

Available to the public from the National Technical Information Service

U.S. Department of Commerce 5285 Port Royal Rd., Springfield, VA 22161 


\title{
A TIME-DEPENDENT BUOYANT PUFF MODEL FOR EXPLOSIVE SOURCES
}

\author{
Edward J. Kansa \\ Earth and Environmental Sciences Directorate \\ Lawrence Livermore National Laboratory \\ Livermore, CA $94551-9900$ \\ email: kansa1@llnl.gov \\ fax: (925)423-6907
}

\begin{abstract}
This paper presents a new model for explosive puff rise histories that is derived from the strong conservative form of the partial differential equations of mass, momenta, and total energy that are integrated over space to yield a coupled system of time dependent nonlinear ordinary differential equations (ODEs). By allowing the dimensions of the puff to evolve laterally and horizontally, the initial rising spherical shaped puff evolves into a rising ellipsoidal shaped mushroom cloud. This model treats the turbulence that is generated by the puff itself and the ambient atmospheric turbulence as separate mechanisms in determining the puff history. The puff rise history was found to depend not only upon the mass and initial temperature of the explosion, but also upon the local stability conditions of the ambient atmosphere through which the puff rises. This model was calibrated by comparison with the Roller Coaster experiments, ranging from unstable to very stable atmospheric conditions; the agreement of the model history curves with these experimental curves was within $10 \%$.
\end{abstract}

\subsection{Introduction}

A two-dimensional or fully three-dimensional time dependent model of buoyant puff rise is impractical in the context of the real time Lawrence Livermore National Atmospheric Response Advisory Capability needs. Consequently, the present approach deals with only volume-averaged quantities that give the time-dependent response of buoyant puffs arising from explosions. This approach reduces the model to a set of coupled, nonlinear ODEs 
that can be integrated very rapidly. A simplified puff-atmospheric turbulence model that is dependent upon the Richardson number (Ri) is developed. This explosive source model is subsequently used to initialize the source in a particle dispersion model.

Baker et al.(1983) define an explosion as a rapid exothermic chemical reaction that produces an overpressure that can be heard. Explosions can be due to either high explosives (e.g, TNT), propellants, or hydrocarbon detonations. High explosives have very short reaction times that produce very hot gases $\left(\mathrm{T}_{\text {expl }} \sim 5,000 \mathrm{~K}\right)$ whereas hydrocarbon explosions are much cooler $\left(\mathrm{T}_{\text {expl }} \sim 1,350 \mathrm{~K}\right)$ and have loner reaction times. The authors present empirical relations for a fireball radius and duration in terms of the mass equivalent of TNT, $M$, as

$$
\begin{aligned}
& \mathrm{R}^{*}=1.93 \mathrm{M}^{0.32} /\left(\mathrm{T}_{\text {expl }} / 3600\right)^{1 / 3} \\
& \mathrm{t}^{*}=0.299 \mathrm{M}^{0.32} /\left(\mathrm{T}_{\text {expl }} / 3600\right)^{10 / 3}
\end{aligned}
$$

While the chemical reaction is proceeding, the spherical volume of radius, $R^{*}$, is assumed to have a uniform burn temperature, $T_{\text {expl }}$. The radius grows at the rate, $R^{*} / t^{*}$ until the time, $t^{*}$ is reached and the chemical reactions cease. Meanwhile, the hot gases rise, expand, entrains and turbulently mix the cool atmospheric air, distort in shape, and eventually rise to a final height in equilibrium with the atmosphere. The equilibrium height to which the puff rises is strongly dependent upon the atmospheric stability condition and will discussed later.

\section{Model Formulation}

The motivation for using the strong conservative form of the Navier-Stokes equations is that the volume integration simplifies by using the Gauss divergence theorem. Since these equations are well known, they will not be stated here. These conservation equations are written in terms of mean and fluctuating variables, e.g. $\rho=\bar{\rho}+\rho ", u=\bar{u}+u^{\prime \prime}$, etc. The mean 
flow is responsible for "entrainment" whereas the fluctuating flow is responsible for turbulent mixing and dissipation. At present, it is assumed that the effects of water vapor are small. (Note that water vapor can be included as an additional conservation equation if considered significant in a particular application.

The interface between the ambient atmosphere and the hot plume is assumed to be a "discontinuity" over which the temperature, density, and velocities vary rapidly. The vertical momentum component is initially zero, but the buoyant hot gas induces a vertical component of momentum upon the existing radial momentum. Thus, the spherical explosion will eventually distort into an idealized elongated ellipsoidal. Many of the previous attempts at modeling puff rise (Turner(1973) and Boughton and DeLaurentis (1987)) constrained the puff to be a sphere throughout the entire rise. The puff appears to be more complicated with the initial spherical cloud evolving into a mushroom-shaped cloud. The present model assumes that the mushroom cap is composed to two half-ellipsoids with a common radius, $r$, in the horizontal plane. The stem is assumed to resemble a cylinder. The upper portion of the cap is a halfellipsoid of height above the center of mass, $\mathrm{z}, \mathrm{h}^{+}$, and the lower portion is an ellipsoid of height, $h^{-}$, below the center, $z$. The center of the cap rises at the velocity, $w_{p}$. Initially, the spherical puff is expanding at a speed, s. By vector addition, the interface at the top and bottom of the puff will experience different net velocities, and gradually, $\mathrm{h}^{+}$and, $\mathrm{h}^{--}$will be distinct. The notation, $\mathrm{h}^{ \pm}$, refers to either $\mathrm{h}^{+}$or $\mathrm{h}^{-}$. The equation for an ellipsoid is:

$\left(\mathrm{x}^{2}+\mathrm{y}^{2}\right) / \mathrm{r}^{2}+\mathrm{z}^{2} /\left(\mathrm{h}^{ \pm}\right)^{2}=1$

The stem can be assumed to be a cylinder of height $\left(z-h^{-}\right)$, and radius, $R^{*}$ Conveniently, such an assumption produces simple algebraic expressions that simplifies the integration over space. The 
stem volume for a cylinder is:

$\mathrm{V}_{\mathrm{s}}=\pi\left(\mathrm{z}-\mathrm{h}^{-}\right) \mathrm{R}^{* 2}$

The ordinary differential equations (ODEs) representing spatially averaged puff conservation of mass, momentum, and total energy are obtained by integrating the governing partial differential equations over space. There are two relevant boundaries: 1. the discontinuity of the hot puff and the ambient atmosphere interface, and 2. the boundary at infinity. At the puff centerline, the flow in the radial direction is zero because of symmetry; likewise, for the gradients in temperature, pressure, and density. Because of entrainment and turbulent mixing, any local gradients within the puff are assumed to rapidly dissipate, the variables within the puff, $\rho_{p}, w_{p}, u_{p}, P_{p}$, and $e_{p}$ are assumed to be spatially uniform. Also, it is assumed that the variables, $\rho_{a}, T_{a}, u_{a}, P_{a}$, and $e_{a}$ are independent of their $x$ and $y$ locations, but only depend on the height, $z$. A simplification can be made by assuming at the height, $z$, that $P_{p}(z)=P_{a}(z)$.

Across the moving puff-atmosphere interface, the jump conditions are be satisfied by using the Liebnitz integration relation at the moving puff-atmosphere interface. The surface areas projected on the radial and vertical directions can be given immediately as:

$\mathbf{n}_{\mathrm{e}} \mathrm{S}_{\mathrm{e}}=\pi\left(2 \mathrm{r}_{\mathrm{e}} \mathrm{h}_{\mathrm{e}} \mathbf{e}_{\mathbf{r}}+2 \mathbf{e}_{\mathrm{k}} \mathrm{r}_{\mathrm{e}}^{2}\right)$

where $e_{r}$ and $e_{k}$ are the unit vectors in the $r$ and $z$ cylindrical coordinates, respectively. The advective fluxes, in the radial and vertical directions, are given by:

$F_{r}=[(\rho u),(\rho u u),(\rho u w),(\rho u e)]^{T}$

and

$F_{z}=[(\rho w),(\rho w u),(\rho w w),(\rho w e)]^{T}$

for the conservation of mass, radial momentum, vertical momentum, and total energy, 
respectively. Because the puff boundary is growing laterally and vertically at some yet unspecified velocity, $\mathbf{s}$,

$\mathbf{s}=\left\{s_{\mathbf{r}} \mathbf{e}_{\mathbf{r}}+s_{\mathrm{z}} \mathbf{e}_{\mathbf{k}}\right\}$

The advective velocity that comprises the flux on either side of the puff interface is shifted in order to coincide with the expanding and distorting interface. The entrainment of a quantity, Q, is given in terms of fluxes of the mean variables. (Note, $Q=1$ when the mass conservation equation is considered.)

The mean value flux quantities are defined as:

$\overline{\mathrm{f}}_{\mathrm{a}}=\bar{\rho}_{\mathrm{a}}\left(\overline{\mathrm{u}}_{\mathrm{a}}-\mathrm{s}_{\mathrm{r}}\right) \mathrm{A}_{\mathrm{r}}+\bar{\rho}_{\mathrm{a}}\left(\overline{\mathrm{w}}_{\mathrm{a}}-\mathrm{s}_{\mathrm{z}}\right) \mathrm{A}_{\mathrm{z}}$

$\bar{f}_{p}=\bar{\rho}_{p}\left(\bar{u}_{p}-s_{r}\right) A_{r}+\bar{\rho}_{p}\left(\bar{w}_{p}-s_{z}\right) A_{z}$

and the turbulent flux quantities as:

$$
\begin{aligned}
& \bar{f}^{\prime \prime}{ }_{a}=\overline{\rho "}{ }_{a} \bar{u}^{\prime \prime} A_{r}+\overline{\rho "} \bar{w}_{a}{ }^{\prime \prime}{ }_{a} A_{z} \text {, } \\
& \overline{\mathrm{f}}_{p}^{\prime \prime}=\bar{\rho}_{p^{\prime \prime}} \bar{u}_{p}{ }_{p} A_{r}+\bar{\rho}_{p} \bar{w}_{p}{ }_{p} A_{z} ;
\end{aligned}
$$

With these preliminary definitions, the well-known strong conservative form of the mass, momenta, and total energy equations are integrated in all three spatial dimensions. using the Liebnitz integral rule, the Gauss divergence theorem, and assuming the mushroom cloud geometry of the explosive puff. The resulting ODEs representing the uniform averaged puff-atmosphere conservation of mass, momentum, and energy are given by:

$$
\begin{aligned}
& \mathrm{d} \overline{\mathrm{m}} / \mathrm{dt}=\left(\overline{\mathrm{f}}_{\mathrm{a}}-\overline{\mathrm{f}}_{\mathrm{p}}\right)+\eta \bar{\rho}_{\mathrm{a}} \mathrm{dV} / \mathrm{dt} \\
& d(\overline{m u}) / d t=\left(f_{a} \bar{u}_{a}-f_{p} \bar{u}_{p}\right)+\left(\bar{f}_{a}^{\prime \prime} \bar{u}_{a}{ }^{-1} \bar{f}_{p}^{\prime \prime} \bar{u}_{p}^{\prime \prime}\right)+\eta \bar{u}_{p} \bar{\rho}_{a} d V / d t \\
& d(\overline{m w}) / d t=\left(\bar{\rho}_{a}-\bar{\rho}_{p}\right) g V+\left(f_{a} \bar{w}_{a}-f_{p} \bar{w}_{p}\right)+\left(\bar{f}_{a}^{\prime \prime} \bar{w}_{a}{ }_{a}-{\overline{f^{\prime \prime}}}_{p} \bar{w}_{p}^{\prime \prime}\right)+\eta \bar{w}_{p} \bar{\rho}_{a} d V / d t, \\
& d(\overline{m e}) / d t=\left(f_{a} \bar{e}_{a}-f_{p} \bar{e}_{p}\right)+\varepsilon \sigma\left(T_{p}^{4}-T_{a}^{4}\right) A_{T}+\left(\bar{\rho}_{a}-\bar{\rho}_{p}\right) g V \bar{w}_{p}+\left(\bar{f}_{a_{a}} \bar{e}^{\prime \prime}{ }_{a}-\bar{f}_{p}{ }_{p} \bar{e}_{p}\right)+\eta \bar{e}_{a} d V / d t
\end{aligned}
$$


where

$m=\int \rho_{p} d V=\bar{\rho}_{p} V$

The rate of entrainment mixing of $\mathrm{Q}$ is limited by the available projected area and difference in fluxes, $\left(f^{a} Q^{a}-f^{p} Q^{p}\right)$. This flux difference tends toward zero as the puff state variables tend toward the ambient atmospheric variables.

The term, $\eta \bar{\rho}_{\mathrm{a}} \mathrm{dV} / \mathrm{dt}$, represents the so-called virtual mass addition term. Daly and Harleman (1966) show that as a body accelerates through a fluid, a force must be applied to that body as well as to the fluid pushed out of the way, and is different from a drag force that is typically dependent upon the square of the relative velocities of an object moving in a fluid. The constant, $\eta$, is about 0.40 to 0.50 .

The combined radial surface area from both the hemisphere and stem are given

as:

$$
\begin{aligned}
& \left.A_{r}=\pi r\left(\left|h^{+}\right|+\left|h^{-}\right|\right)+2 \cdot \pi r^{2} z^{2} /\left(r^{2}+z^{2}\right)\right), \\
& A_{z}=2 \pi r^{2}+\pi r z\left(2 r /(r+z)-r z /\left(r^{2}+z^{2}\right)\right) .
\end{aligned}
$$

Because the puff cap is assumed to be the union of two halves of an ellipsoid, the time rate of change for the puff heights in terms of the radial growth rate is given by:

$\mathrm{dh}^{+} / \mathrm{dt}=-\left(\mathrm{h}^{+} / \mathrm{r}\right) \mathrm{dr} / \mathrm{dt},\left(\mathrm{h}^{+}>0\right)$

$\mathrm{dh}^{-} / \mathrm{dt}=-\left(\mathrm{h}^{-} / \mathrm{r}\right) \mathrm{dr} / \mathrm{dt},\left(\mathrm{h}^{-}<0\right)$

Because $\mathrm{h}^{+}$decreases in time as $\mathrm{r}$ grows, $\mathrm{h}^{+}$is constrained to always be greater than a cutoff value taken to be $2 \mathrm{~m}$. By manipulating the time rates of change of mass, volume, and the conservation of mass equations, the radial component of the moving frame velocity is given by

$s_{r}=3\left\{2\left(\bar{\rho}_{\mathrm{a}} \bar{u}_{\mathrm{a}}-\bar{\rho}_{\mathrm{p}} \overline{\mathrm{u}}_{\mathrm{p}}\right)\right\} /\left\{\left[(6-8 \eta) \bar{\rho}_{\mathrm{a}}+2 \bar{\rho}_{\mathrm{p}}\right]\right\}$. 
and

$\mathrm{s}_{\mathrm{z}}^{ \pm}=-\left(\mathrm{h}^{ \pm} / \mathrm{r}\right) \mathrm{dr} / \mathrm{dt}$

\section{Turbulence Models}

The turbulent eddy viscosity and thermal conductivity are assumed to have two contributions: one arising from the ambient atmosphere, and the other from the puff itself. It is assumed that the local Richardson number, $\mathrm{Ri}$, characterizes the turbulence in both the atmosphere and puff. The ambient turbulent atmosphere can be treated by standard methods derived from measured quantities and a variety of stability conditions. The atmospheric turbulence contribution, $\overline{\mathrm{f}^{\prime \prime}} \bar{a}_{\mathrm{w}^{\prime \prime}}{ }_{\mathrm{a}}, \overline{\mathrm{f}^{\prime \prime}}{ }_{\mathrm{a}} \overline{\mathrm{u}}^{\prime \prime}{ }_{\mathrm{a}}$, and $\overline{\mathrm{f}}_{\mathrm{a}}{ }_{\mathrm{e}} \overline{\mathrm{e}}_{\mathrm{a}}$ appearing in Eqs $(12-15)$ uses the atmospheric turbulence models at various layers presented by Pielke (1984). The local static stability is determined by the local lapse rate is misleading and often fails in the convective mixed layer. The rise of thermals from the surface or their descent from cloud tops depends on their excess buoyancy, not the ambient lapse rate. Stability must be determined with respect to water vapor content and the saturated adiabats. The virtual potential temperature is defined as

$\theta_{v}=T_{v}+0.00981 * z$

where

$T_{v}=T(1+0.61 q)$.

and $\mathrm{q}$ is the specific humidity (the mass of water vapor per unit mass of air). Following Fleagle and Businger (1980), the non-dimensional Richardson number, $\mathrm{Ri}$, which denotes the ratio of the buoyant energy to the shear kinetic energy, and is defined as:

$\mathrm{Ri}=\mathrm{g} \partial \ln \theta_{\mathrm{v}} / \partial \mathrm{z} /\left[(\partial \mathrm{u} / \partial \mathrm{z})^{2}+(\partial \mathrm{v} / \partial \mathrm{z})^{2}\right]$

Fleagle and Businger (1980) classify the atmosphere as unstable (convective) for $\mathrm{Ri}<0$, neutral for $\mathrm{Ri}=0$, and stable for $\mathrm{Ri}>0$. 
In contrast to the well-studied atmospheric turbulence, the puff-atmospheric turbulence model is an uncharted area. Libby and Williams (1980) have pointed out that clouds of either hot or cold gases can enhance or suppress turbulent mixing. The puff-atmospheric turbulence model involves those fluctuating terms having the following structure $A_{i}\left[\overline{\rho^{\prime \prime}} \bar{Q}_{p}{ }_{p} \bar{w}_{p}\right]$

All turbulence models require a means of closure. The puff-atmospheric interface is quite turbulent, so any particle at or near this edge may be inside or outside of the puff at any instant of time. To avoid calculating very detailed complex higher order closure models, the following assumptions were made:

$\bar{\rho}_{p}^{\prime \prime}=\bar{\rho}_{p}-\bar{\rho}_{\mathrm{a}}$,

$\overline{\mathrm{Q}}_{\mathrm{p}}^{\prime \prime}=\overline{\mathrm{Q}}_{\mathrm{p}}-\overline{\mathrm{Q}}_{\mathrm{a}}$,

$\bar{w}_{p}=\bar{w}_{p}$ (assuming $\bar{w}_{\mathrm{a}}=0$ )

$\bar{u}_{p}=\bar{u}_{p}-\bar{u}_{a}$

$\overline{\mathrm{e}}_{\mathrm{p}}=\overline{\mathrm{e}}_{\mathrm{p}}-\overline{\mathrm{e}}_{\mathrm{a}}$

The contribution to the puff-atmosphere projected on the $\mathrm{i}$-th axis is given by:

$A_{i}\left[\bar{\rho}_{p}{ }_{p} \bar{u}_{i, p} \bar{u}_{j p}\right]=k_{p-a} A_{i}\left(\bar{\rho}_{a}-\bar{\rho}_{p}\right)\left(\bar{u}_{i, p}-\bar{u}_{i, a}\right)\left(\bar{u}_{j, p}-\bar{u}_{j, a}\right)$

$A_{i}\left[\bar{\rho}_{p}{ }_{p} \bar{e}_{p} \bar{u}_{i, p}\right]=k_{p-a}^{e} A_{i}\left(\bar{\rho}_{p}-\bar{\rho}_{a}\right)\left(e_{p}-\bar{e}_{a}\right)\left(\bar{u}_{i, p}-\bar{u}_{i, a}\right)$

where $A_{i}$ is either the $r$ or $z$ projected area, $k_{p-a}$ and $k_{p-a}^{e}$ are the effective eddy viscosity and turbulent thermal conductivities, respectively. These constants, $\mathrm{k}_{\mathrm{p}-\mathrm{a}}$ and $\mathrm{k}_{\mathrm{p}-\mathrm{a}}^{\mathrm{e}}$, were determined empirically by comparing the model results with the observed puff height of the Roller Coaster experiments, Church (1969). For simplicity, it was assumed that $\mathrm{k}_{\mathrm{p}-\mathrm{a},}^{\mathrm{e}}=\mathrm{k}_{\mathrm{p}-\mathrm{a}}$.

Note that these turbulence terms in the two momentum equations involve 
products of the velocity differences, and can be interpreted as "turbulent drag" terms. These "drag" terms render the system of ODEs nonlinear. However, unlike the atmospheric turbulence, the puff-atmospheric turbulence terms, Eqs(31-32) vanish when the puff dependent variables approach atmospheric values; this property is consistent with the assumption made earlier. Entrainment, the atmospheric turbulence, and puff-atmospheric turbulence are considered to be the dissipation terms whose magnitudes determine the time at which the puff reaches a steady state height.

Atmospheric stability plays a very important role in governing the rate of rise of the puff and in determining the time required for a puff to reach a steady state height. The puffatmospheric dissipation terms, $\mathrm{k}_{\mathrm{p}-\mathrm{a}}$ and $\mathrm{k}_{\mathrm{p}-\mathrm{a}}^{\mathrm{e}}$, are assumed to be a function of the local $\mathrm{Ri}$, and height, $z$, above the surface, similar to the atmospheric turbulence models. The height of the surface and mixed layers is a function of time of day, degree of surface heating, surface type, albedo, soil moisture and latitude. In summer months, the mixed layer characterized by strong convective heating can be anywhere from 1,000 to $2,000 \mathrm{~m}$ above the surface. On clear nights, near dawn, the convective mixing of the atmosphere ceases, and the atmosphere is very stable with the mixing depth from 100 to $300 \mathrm{~m}$ above the surface. The above model is convenient to characterize the various atmospheric stability conditions. The present puff rise model assumes that the atmospheric soundings are available to determine the initial conditions.

An eddy diffusivity coefficient, $\mathrm{k}_{\mathrm{p}-\mathrm{a}}(\mathrm{z})$, is assumed to be a function of height, $\mathrm{z}$. From the surface to the top of the surface layer, $\mathrm{k}_{\mathrm{p}-\mathrm{a}}$ is assumed to vary linearly with $\mathrm{z}$. From the surface layer to the transition height

$z_{\text {transition }}=z_{\mathrm{sl}}+\left(\mathrm{z}_{\mathrm{ml}}-\mathrm{z}_{\mathrm{sl}}\right) / 3$

$\mathrm{k}_{\mathrm{p}-\mathrm{a}}$ becomes a maximum at $\mathrm{z}_{\text {transition }}$, and then reduces to a smaller value at $\mathrm{z}=\mathrm{z}_{\mathrm{ml}}$. Above $\mathrm{h}_{\mathrm{ml}}$, 
$\mathrm{k}_{\mathrm{p}-\mathrm{a}}$ is assumed to be constant.

In general, a puff that has lost much of its buoyant momentum can be trapped at the mixed layer. The critical feature determining whether a puff will be trapped at this layer, or punch through it, depends upon the excess vertical momentum of a puff. For a stable atmosphere, an explosion with a certain mass will equilibrate at the mixed layer, but an explosion with double the mass may punch through and equilibrate at a much larger height. Because of the initial temperature difference, an exploding gasoline vapor cloud may equilibrate at a much lower height than a TNT explosion.

By experimentation, the puff-atmospheric eddy coefficients were found to increase with increasing $\mathrm{Ri}$ whereas the ambient atmospheric turbulence decreases with increasing $\mathrm{Ri}$. In the unstable atmospheric conditions, the ambient atmospheric eddy coefficients increased with decreasing $\mathrm{Ri}$, and the puff-atmospheric eddy coefficients decreased with decreasing $\mathrm{Ri}$. At present, there has not been any detailed studies or models formulated to understand the turbulence of hot gases mixing with the cooler atmosphere. The determination of the puff-atmospheric turbulence parameters is basically an inverse problem.

Church (1969) has reported the measured puff cloud top heights as a function of time for varying amounts of explosive and atmospheric stability conditions. Ideally, the inverse problem could be solved by time marching the set of nonlinear ODEs of the present model simultaneously and adjusting the parameters simultaneously to minimize the least squares errors between the predicted puff cloud heights and the observed heights. However, such an approach requires huge demands upon the computer resources, and a simpler approach was used. Rather each experiment was optimized separately to obtain a reasonable average of the parameters. The experiments used from 6.4 to $1019 \mathrm{~kg}$ TNT equivalent amounts of explosive mass. In a sense, 
the parameters were interpolated for this range of TNT explosive. Whether such parameters are reasonably valid beyond range for which they were calibrated can only be determined by future experiments. In addition, there is a concern that three dimensional effects such as large atmospheric shear can become quite important for very large explosions. Such effects were ignored in order to obtain such a simple model.

Table 1 lists the maximum and minimum values for the puff-atmospheric eddy viscosity and area multipliers. Model experimentation revealed that for very stable atmospheres, the puff-atmospheric viscosity and area multiplicative factors needed to be increased with increasing positive Ri number, and visa versa. Intuitively, this is physically consistent. An unstable atmosphere is very turbulent, and in such an atmosphere, the puff is being buffeted by strong eddies that induce mixing rapidly, so the puff-generated turbulence is of minor importance. However the presence of finite duration updrafts or downdrafts can either accelerate or decelerate the puff dramatically. For neutral or stable conditions, the atmospheric turbulence plays a progressively decreasing role with increasing positive $\mathrm{Ri}$ number. In very stable atmospheres with $\mathrm{Ri}>0.5$, the puffs are observed to equilibrate rapidly to the mixed layer that can be relatively close to the surface. Thus, the hot puff must dissipate its heat rapidly. This implies that entrainment and the puff generated turbulence are the dominant mechanisms. So for increasing positive $\mathrm{Ri}$, the effective area and puff eddy dissipation would increase with $\mathrm{Ri}$. In Table $1, \mathrm{k}_{\mathrm{p}-\mathrm{a}}=\mathrm{k}_{\mathrm{p}-\mathrm{a}}^{\mathrm{e}}$ are listed as a maximum and minimum values for the various ambient atmospheric Richardson numbers. Likewise, the effective area coefficient, $\Xi$, is assumed to vary with height similar to the puff-atmospheric eddy viscosity coefficients. These parameters were fine-tuned to the $63.6 \mathrm{~kg}$ TNT equivalent of the Roller Coaster experiments. In a separate 
section, these parameters will be adjusted for larger or smaller explosions.

Table 1: Puff-atmospheric parameters calibrated for $63.6 \mathrm{~kg}$ TNT explosions

\begin{tabular}{|l|l|l|l|l|l|l|}
\hline Attribute & $-\mathbf{0 . 5}<\mathbf{R i}<-\mathbf{0 . 1}$ & $-\mathbf{0 . 1} \leq \mathrm{Ri}<\mathbf{0}$ & $\mathbf{0 . 0 \leq R i < 0 . 5}$ & $\mathbf{0 . 5} \leq \mathrm{Ri}<\mathbf{0 . 8}$ & $\mathbf{0 . 8} \leq \mathrm{Ri} \leq \mathbf{1 . 0}$ & $\mathbf{R i}>\mathbf{1 . 0}$ \\
\hline $\mathbf{k}_{\mathrm{p}-\mathbf{a}}(\mathbf{m a x})$ & 0.08 & 0.090 & 0.16 & 1.09 & 2.05 & 5.0 \\
\hline $\mathbf{k}_{\mathbf{p}-\mathbf{a}}(\min )$ & 0.06 & 0.075 & 0.10 & 1.05 & 1.70 & 1.75 \\
\hline$\Xi(\max )$ & 0.50 & 0.53 & 1.03 & 1.10 & 1.20 & 2.25 \\
\hline$\Xi(\min )$ & 0.40 & 0.42 & 0.98 & 1.02 & 1.03 & 1.5 \\
\hline
\end{tabular}

This table illustrates that the mean flow entrainment and "turbulent drag" upon the hot puff increases with increasing $\mathrm{Ri}$. This is physically consistent with the experimental observations that a puff looses its excess buoyancy rapidly in a stable atmosphere, but rises higher in a less stable atmosphere. In seperate model calculations, it was observed that for a very stably layered atmosphere, that doubling the explosive mass causes the puff to equilibrate at a significantly higher layer, while halving the explosive mass causes the puff to equilibrate at a lower height. Similarly, if the lower layers of the atmosphere are unstable, say for the first kilometer above the surface, the standard atmospheric model of the upper portions of the atmosphere tend to have more neutral atmospheric conditions. So a puff generated by a rather large explosive mass rising in the upper layers will entrain cooler atmospheric air and encounter a "turbulent drag" that eventually slows and stops a puff from rising further.

\section{Approximate Analytic Solutions}

The various Roller Coaster and Site 300 experiments were integrated numerically because the puff dimensions and density, are constantly changing dimensions and exact solutions are intractable. It is convenient to examine simplifying analytic expressions to obtain a "feel" for the physics involved. Assume that in a small time interval, $\Delta t$, the puff mass, $m_{p}$, the 
density difference term, $\Delta \bar{\rho}$, the puff dimensions $s_{r}$, the interface velocities, $s_{r}$ and $s_{z}$, and the turbulence parameters, $\mathrm{k}_{\mathrm{p}-\mathrm{a}}$ and $\mathrm{k}_{\mathrm{p}-\mathrm{a}}^{\mathrm{e}}$, are slowly varying.

For convenience, the atmospheric turbulence is defined as

$\Lambda_{\mathrm{atm}}=-\mathrm{K}_{\mathrm{atm}}(\mathrm{Ri})\left(\partial \bar{u}_{\mathrm{a}} / \partial \mathrm{z}\right)$

and $\mathrm{F}^{\prime}=\mathrm{F}_{\mathrm{a}}+\Delta \overline{\mathrm{\rho}} \mathrm{k}_{\mathrm{p}-\mathrm{a}} \mathrm{A}_{\mathrm{r}} \Delta \mathrm{u}$.

A rearrangement of the vertical velocity ODE gives

$d w_{p} /\left(\Delta \bar{\rho} k_{p-a} A_{z} \bar{w}_{p}^{2}+F \bar{w}_{p}+\left(\Lambda_{a t m}-\Delta \bar{\rho} g V\right)+d t / M_{p}=0\right.$

Under the above assumption, this ODE can be integrated to yield the following expression:

$\bar{w}_{p}=\left[\delta-F^{\prime}+\left(\delta+F^{\prime}\right) * \exp \left(-\delta \Delta t / M_{p}\right)\right] /\left\{\left[1-\kappa_{o} \exp \left(-\delta \Delta t / M_{p}\right)\right] \Delta \bar{\rho} k_{p-a} A_{z}\right\}$

where $\delta=\left[\left(\mathrm{F}^{\prime}\right)^{2}-4\left(\Delta \bar{\rho} \mathrm{gV}-\Lambda_{\mathrm{atm}}\right)\left(\Delta \bar{\rho} \mathrm{k}_{\mathrm{p}-\mathrm{a}} \mathrm{A}_{\mathrm{z}}\right)\right]^{1 / 2}$.

and $\kappa_{0}=-\log \left(\delta+F^{\prime}\right)$.

Assume $0<\Delta \bar{\rho} \leq 0.5 \bar{\rho}_{\mathrm{a}}$. The expression for $\mathrm{w}_{\mathrm{p}}$ simplifies, after expanding $\delta$ to yield:

$\bar{w}_{p} \cong\left[2\left(\Delta \bar{\rho} \mathrm{gV}-\Lambda_{\mathrm{atm}}\right) / \mathrm{F}^{\prime}+\mathrm{F}^{\prime} \exp \left(-3 \overline{\mathrm{u}}_{\mathrm{a}} \Delta \mathrm{t} / \mathrm{h}\right)\right] /\left(\left[1-\kappa_{\mathrm{o}} \exp \left(-3 \overline{\mathrm{u}}_{\mathrm{a}} \Delta \mathrm{t} / \mathrm{h}\right)\right]\right.$

The buoyant velocity, $w_{p}$, is directly proportional to the difference of the buoyant force and atmospheric dissipation, and inversely proportional to the surface area and wind velocity. The greater the ambient wind speed, $\bar{u}_{\mathrm{a}}$, the faster $\overline{\mathrm{w}}_{\mathrm{p}}$ decays. Next, assume that the radiation transport is small; the energy equation can be linearized. For convenience, define:

$F^{\prime \prime}=\left\{F_{p}+k_{p-a}^{e}\left(\bar{\rho}_{a}-\bar{\rho}_{p}\right)\left\{A_{r}\left(u_{p}-u_{a}\right)+A_{z}\left(w_{p}-w_{a}\right)\right\}\right]$

and $G=\left(\bar{\rho}_{\mathrm{a}}-\bar{\rho}_{\mathrm{p}}\right) \mathrm{gVw} \mathrm{w}_{\mathrm{p}}-\mathrm{K}_{\mathrm{e}}^{\mathrm{atm}}-\varepsilon \sigma\left(\mathrm{T}_{\mathrm{p}}^{4}-\mathrm{T}_{\mathrm{a}}^{4}\right) \mathrm{A}_{\mathrm{T}}$.

The solution to the differential equation

$d e_{p} / d t+\left(F^{\prime \prime}\left(e_{p}-e_{a}\right)+G\right)=0$ 
is $e_{p}=e_{a}+\left(\kappa_{e} \exp \left(-\delta^{\prime} \Delta \tau / M_{p}\right)-G\right) / F^{\prime \prime}$

. As with the expression for $w_{p}$, increasing the wind speed decreases the time for the energy of the puff to approach the ambient energy at a height, $z$. Also, the larger the mass of the puff, the longer is the time required the puff energy to dissipate. A necessary and sufficient condition for stable, physically acceptable solutions is that the puff turbulence terms be positive definite. From these simplified expressions, it is clear that for sufficiently long time, there exists a height $H$ such that $w_{p}(H) \rightarrow 0, u_{p}(H) \rightarrow u_{a}(H), \rho_{p}(H) \rightarrow \rho_{a}(H)$, and $T_{p}(H) \rightarrow T_{a}(H)$.

\section{Roller Coaster Series}

The model results were primarily compared and calibrated against the series of Roller Coaster high explosive experiments undertaken at Tonopah Test Range at the Nevada Test Site in May and June, 1963. These shots were performed from dusk to dawn under a variety of atmospheric stability conditions, ranging from unstable to very stable atmospheric conditions. The height of the cloud tops was obtained either by photographs or by theodolite tracking measurements. A vertical illuminated grid was erected to measure photographically the puff tops at various times, up to 5 minutes after the explosion. The ambient temperature and wind profiles were measured up to $600 \mathrm{~m}$ from the surface at a station $3.6 \mathrm{~km}$ from ground zero. These data and the observed heights at various times were documented by Church (1969) and were used to construct the local Ri number profile for each experiment.

Figure 1 shows the puff height histories for unstable, neutral, and stable atmospheric conditions for constant $63.6 \mathrm{~kg}$ TNT equivalent explosions. Figure 2 shows two puff height histories under stable conditions, but with two different TNT mass equivalents. Upon examining the plots, most of the errors resulted from the mismatch at early times. However, the asymptotic time heights are within $5 \%$ of the measured heights, denoted by the $x$ 's in these figures. 


\section{Statistical analysis of the model predictions against the Roller Coaster experiments}

The absolute residual error were calculated using measured 12 Roller Coaster and 3 Clean Slate field experiments against the prediction of the present model. Church (1969) listed the heights of the cloud tops at the following intervals: $0.5,1.0,2.0,3.0,4.0$, and 5.0 minutes after detonation. The average absolute residual error (AARE) is defined as:

AARE $\left.=\Sigma_{t=1} \mid z_{\text {model }}\left(t_{i}\right)-z_{\text {field }}\left(t_{i}\right)\right) \mid l /\left(z_{\text {field }}\left(t_{i}\right) N_{\text {obs }}\right]$

The average absolute residual error over the entire set of Roller Coaster and Clean Slate experiments is about a $10.1 \%$. The predicted early time results account for most of the error.

\section{Correlation of Parameters As a Function of Explosive Mass}

As stated previously, a very important parameter determining the eventual equilibrium puff cloud height is the TNT mass equivalent of the explosive. For a given atmospheric stability, the greater the explosive mass, the greater will be the time to achieve equilibrium and the higher will be the steady state height be, see Figure 2. In general, the lower layers of the atmosphere can experience a wide range of Richardson numbers, but above the boundary layer whose height can also vary considerably.

The experiments against which this model was calibrated had TNT equivalent masses ranging between $63-1020 \mathrm{~kg}$, and the maximum time for which measurements were taken was 300 seconds after the ignition time. An attempt was made to correlate the mass of the explosive to parameters multiplying the puff-atmospheric turbulence parameter, $\mathbf{k}_{\mathbf{p - a}}$, and the effective cross-sectional area, $\Xi$, as functions of explosive mass and average Richardson number up to the boundary layer.

Denote constants, $C_{1}$ and $C_{2}$ as input constants that multiply $\mathbf{k}_{\mathbf{p}-\mathbf{a}}$, and $\Xi$, 
respectively. Since the most prevalent TNT mass in the Roller Coaster series was $63.6 \mathrm{~kg}$ of TNT, correlations of TNT mass, denoted by $M$ were made.

Table 2: Parameters $C_{1}$ and $C_{2}$ dependence on explosive mass, $M$

\begin{tabular}{|l|l|l|}
\hline \multicolumn{1}{|c|}{ Atmospheric Stability } & \multicolumn{1}{c|}{$\mathrm{C}_{1}$} & \multicolumn{1}{c|}{$\mathrm{C}_{2}$} \\
\hline \hline Unstable atmosphere & 0.19 & 0.37 \\
\hline Neutrally Stable atmosphere & 0.40 & 0.5 \\
\hline Mildly Stable atmosphere & $0.5-0.15 \log _{10}(\mathrm{M} / 63.6)$ & 0.6 \\
\hline Very Stable atmosphere & $0.6-0.34 \log _{10}(\mathrm{M} / 63.6)$ & $0.6+0.094 \log _{10}(\mathrm{M} / 63.6)$ \\
\hline
\end{tabular}

For the parameters, $C_{1}$ and $C_{2}$, a minimum value of the unstable atmosphere should be used for very large explosions. Because the model was calibrated with a limited set of experiments, extrapolation to explosive masses beyond the calibration limits should be used with caution.

\section{Summary}

A buoyant puff rise model was developed from the strong conservative form of the Navier-Stokes equations by integrating over space to yield a set of coupled nonlinear ODEs dependent upon the jump conditions of mass, momentum, and energy at the puff-atmosphere interface. The puff cloud was allowed to evolve from a sphere to an ellipsoid by allowing lateral and vertical degrees of freedom evolving into a mushroom shaped cloud. It was found that the rise history of an explosive cloud depended upon not only the explosive mass and temperature, but also upon the strength of the puff-atmospheric turbulence, the wind speed, and the effective cross-sectional areas. The coupled nonlinear ODEs represented in the model are integrated in a matter of a few seconds of computer time.

The buoyant rise of the hot mushroom cloud is dissipated by radiation, entrainment from the mean flow, puff-atmospheric turbulence, and the ambient turbulence. 
From model experimentation, the ambient and puff-atmospheric turbulence were observed to affect the rate at which a puff rises. From the limited number of model comparisons to field experiments, the model produced excellent agreement with actual puff rise histories. It is observed that slugs of hot gas has its own turbulence properties as the slug rises in the ambient atmosphere. The atmosphere is very turbulent in the unstable $(\mathrm{Ri}<0)$ region, the puffatmosphere turbulence diffusivities are quite small. In the very stable atmospheres $(\mathrm{Ri}>1)$, the atmospheric diffusivities are negligible, but the puff-atmosphere turbulence diffusivities are quite large. It would be very desirable to obtain a better understanding of this phenomenon.

At least for the Roller Coaster, Clean Slate, and Site 300 experiments, the calibrated model puff height history curves were within $10 \%$ error of the experimentally measured results with the largest errors occurring at the early times. Although the spatial dependence are integrated out, it can be concluded that this model appears to have captured the essence of the most important mechanisms describing the buoyant puff rise histories, at least for the Roller Coaster and Site 300 experiments.

\section{Acknowledgments}

This work was performed under the auspices of the U.S. Department of Energy by Lawrence Livermore National Laboratory under Contract No. W-7405-Eng.48

\section{Keywords}

buoyant puff rise history, explosives, turbulence, atmospheric stability

\section{References}

Baker,W.E., Cox, P.A. Westine, P.S., Kulesz, J.J, and Strehlow, R.A. (1983), Explosion Hazards and Evaluation, Elsevier Scientific Publ. Co, NY. 
Boughton, B.A. and DeLaurentis (1987), An integral model of plume rise from high explosive detonations, 70: 27-32. ASME-HTD 24th Annual National Heat Transfer Conference.

Church, H.W, (1968), Cloud Rise from High Explosive Detonations, SC-RR-68-903, Sandia National Laboratory.

Daly, J.W. and Harleman, D.R.F (1966), Fluid Dynamics, Addison-Wesley Publ. Reading, MA.

Fleagle, R.G. and Businger, J.A. (1980), An Introduction to Atmospheric Physics, Academic Press, Inc. NY.

Libby, P.A. and Williams, F.A. (1980), Turbulent Reacting Flows, Springer Verlag, Berlin; New York.

Pielke, R.A. (1984), Mezoscale Meteorological Modeling, Academic Press, New York.

Turner, J.S. (1973), Buoyancy Effects in Fluids, Cambridge University Press. 


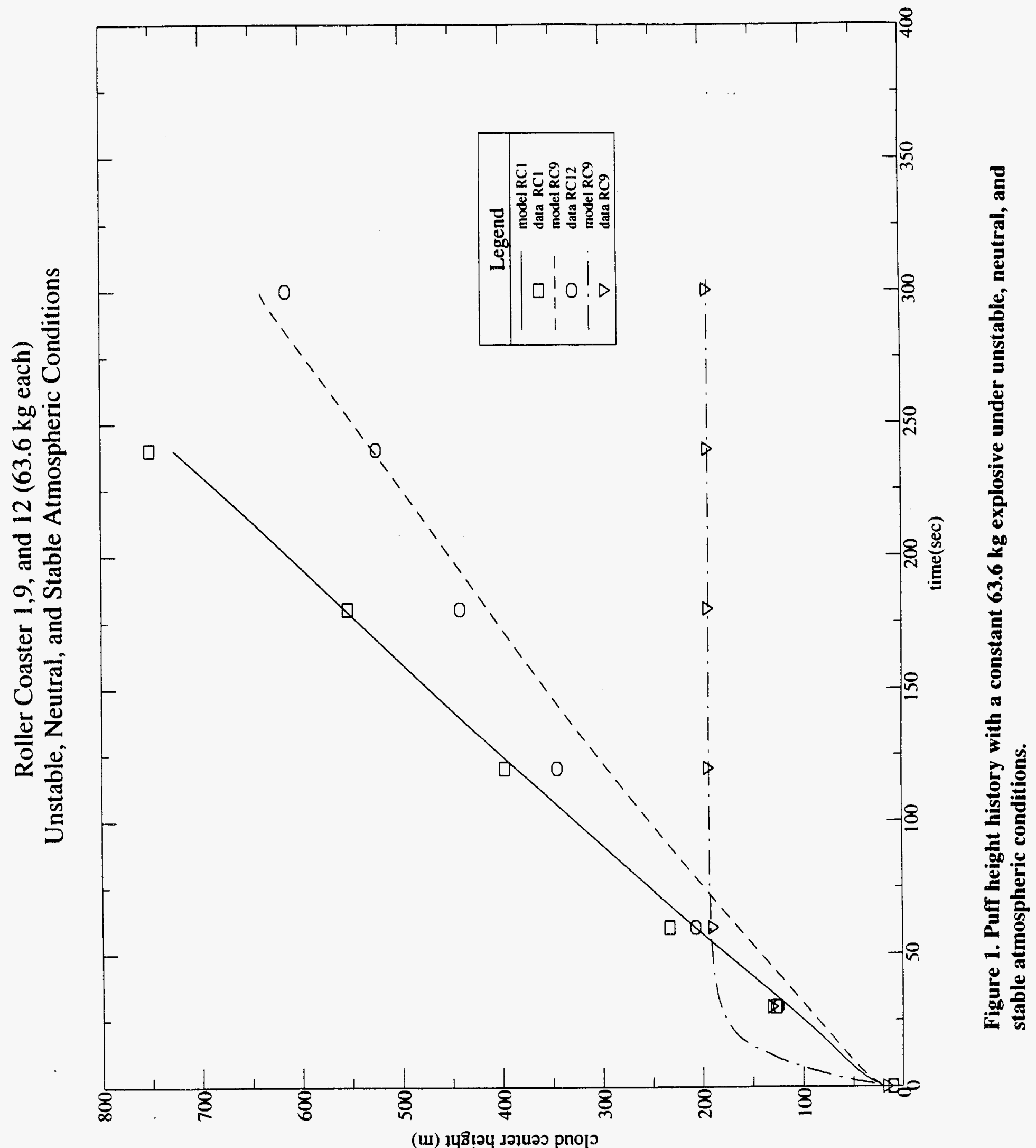




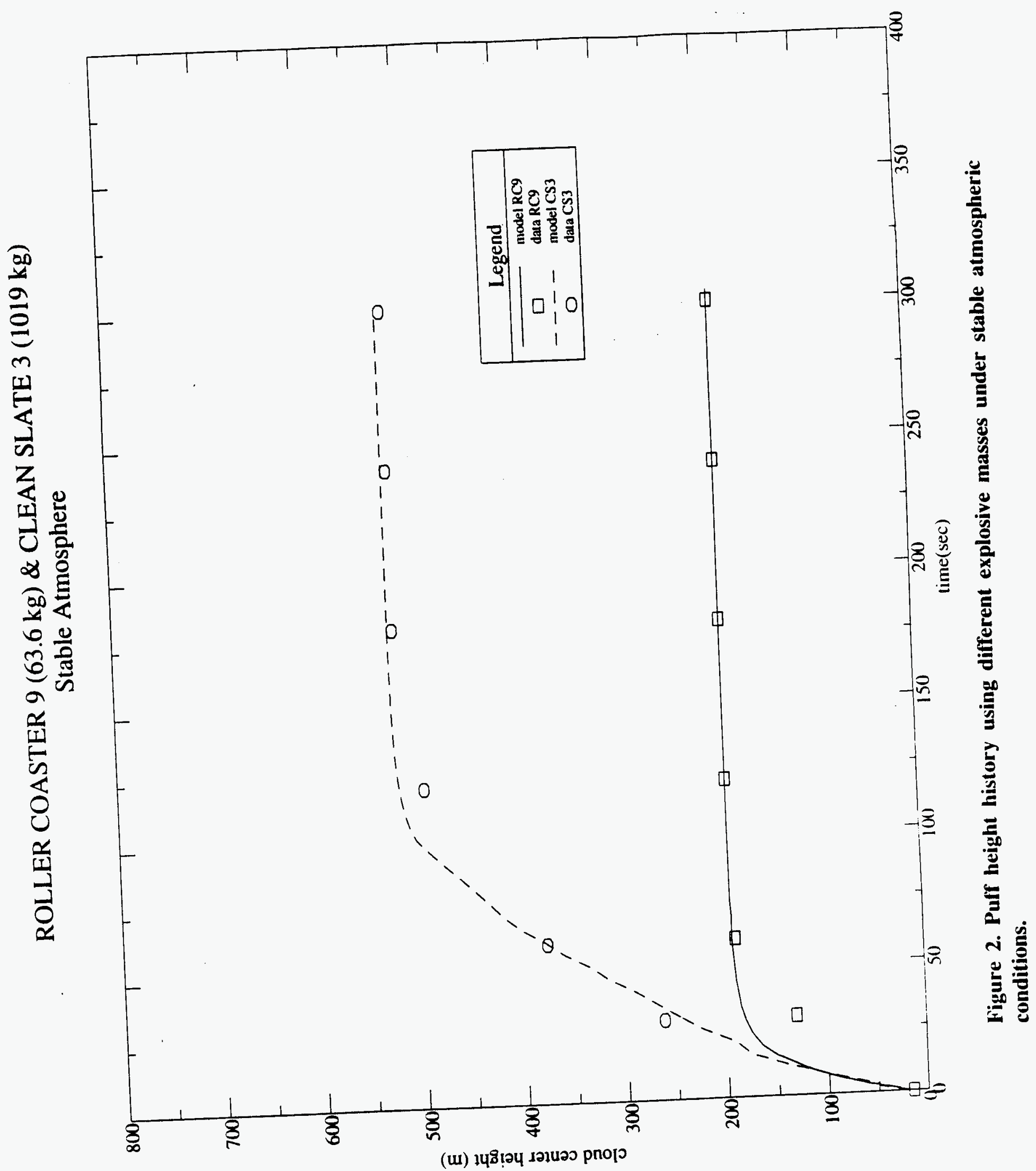


Technical Information Department • Lawrence Livermore National Laboratory University of California • Livermore, California 94551

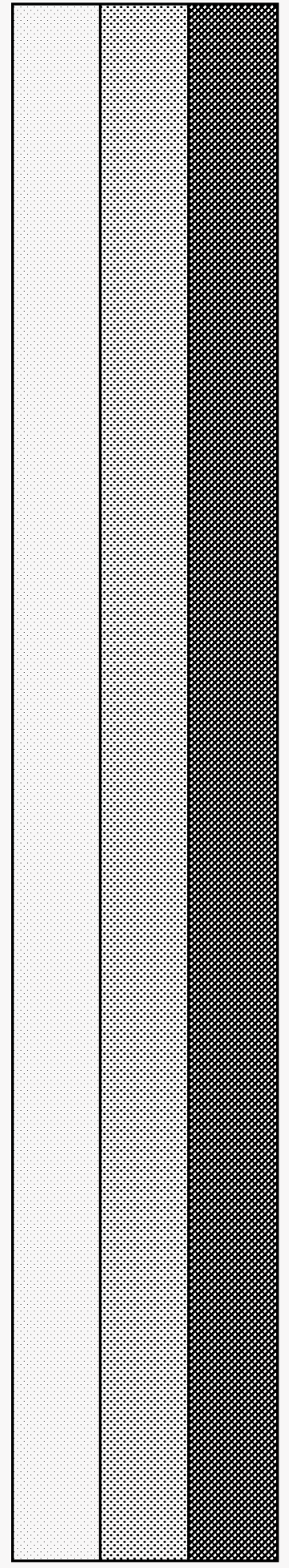

